\title{
PSMA PET/CT to evaluate response to SBRT for prostate cancer bone metastases
}

\author{
Igor Sadetski ${ }^{1 \star}$, Yael Eshet ${ }^{2 \star}$, Orit Kaidar-Person ${ }^{(1}{ }^{1}$, Uri Amit $^{1}$, Liran Domachevsky ${ }^{2}$, Tima Davidson ${ }^{2}$, \\ Ilana Weiss ${ }^{1}$, Maoz Ben Ayun ${ }^{1}$, Zvi Symon ${ }^{1 *}$ \\ ${ }^{1}$ Radiation Oncology, Chaim Sheba Medical Center, affiliated with the Sackler School of Medicine, Tel Aviv University, Israel \\ ${ }^{2}$ Nuclear Medicine, Chaim Sheba Medical Center, affiliated with the Sackler School of Medicine, Tel Aviv University, Israel \\ ${ }^{\star}$ The first, second and last authors contributed equally to this publication
}

\begin{abstract}
Background: In the current study we evaluated ${ }^{68} \mathrm{Ga}$ PSMA PET/ CT to measure local control of bone metastasis in oligometastatic prostate cancer patients treated with SBRT.

Materials and methods: After the institutional review board approval, a retrospective review of medical records of consecutive prostate cancer patients treated between 2014 and 2018 was conducted. Only medical records of patients that were treated with SBRT for bone metastasis and had pre-and post-SBRT ${ }^{68} \mathrm{Ga}$ PSMA PET/CT scans were included in our study. Data extracted from the medical files included patient-related (age), disease-related (Gleason score, site of metastasis), and treatment-related factors and outcomes.

Results: During the study period, a total of 12 patients ( 15 lesions) were included, with a median age of 73 years. The median follow-up was 26.5 months (range 13-45 months). Median time of ${ }^{68} \mathrm{Ga}$ PSMA PET/ CT follow up was 17.0 months (range 3-39 months). The median pre-treatment PSA was $2 \mathrm{ng} / \mathrm{mL}$ (range $0.56-44 \mathrm{ng} / \mathrm{mL}$ ) vs. post treatment PSA nadir of $0.01 \mathrm{ng} / \mathrm{mL}$ (0.01-4.32) with a median time to nadir of 7 months (range, 2-12). Local control was $93 \%$ during the follow up period and there was correlation with PSMA avidity on PET. None patients developed recurrences in the treated bone. None of the patients had grade 3 or more toxicities during follow-up.

Conclusions: SBRT is a highly effective and safe method for treatment of prostate cancer bone metastases. More studies are required to determine if SBRT provides greater clinical benefit than standard fractionation for oligometastatic prostate cancer patients. ${ }^{68} \mathrm{Ga}$ PSMA PET/CT should be further investigated for delineation and follow-up.
\end{abstract}

Key words: PSMA; PET; prostate; radiation; stereotactic; SBRT; cancer

Rep Pract Oncol Radiother 2021;26(4):528-534

\section{Introduction}

Oligometastastic disease is assumed to be an expression of biological indolent metastatic disease, that is a low burden and has little effect on patient's performance status. However, there is no consensus on the definition or means needed to diagnose these favorable metastatic patients [1]. The optimal management of oligo-recurrent prostate cancer is also an ongoing area of controversy. The traditional treatment approach for favorable metastatic disease has been conservative and favored observation and then systemic or palliative therapy [2]. Low burden disease among other considerations, including ad-

Address for correspondence: Zvi Symon, MD, Associate Professor and Chair Radiation Oncology, Sheba Medical Center, Ramat Gan, Tel Hashomer, Israel, Sackler School of Medicine, Tel Aviv University; e-mail: Zvi.Symon@sheba.health.gov.il

This article is available in open access under Creative Common Attribution-Non-Commercial-No Derivatives 4.0 International (CC BY-NC-ND 4.0) license, allowing to download articles and share them with others as long as they credit the authors and the publisher, but without permission to change them in any way or use them commercially 
vances in imaging and radiation therapy (RT) delivery, led to the increasing use of ablative treatments to these patients. The SABR-COMET trial [3], a phase II trial ( $21 \%$ prostate cancer) showed a statistically significant survival advantage for stereotactic ablative radiation (median overall survival, 41 months versus 28 months in the control group) indicates that ablative approach for oligometastatic disease should be further explored.

The yield of imaging and early detection of metastatic disease on outcome is still unclear. New Positron Emission Tomography (PET) tracers provide opportunities to detect oligometastases in prostate cancer patients, both at initial cancer diagnosis and at the time of biochemical failure following treatment [4].

Prostate-Specific Membrane Antigen (PSMA) is a type II transmembrane protein and is typically overexpressed in prostate cancer tissue. ${ }^{68}$ Gallium $\left({ }^{68} \mathrm{Ga}\right)$ PSMA PET/Computed Tomography (CT) has high sensitivity for the detection of low burden metastatic disease [4].

Stereotactic body radiotherapy (SBRT) is an appealing treatment option for metastasis-directed therapy given its non-invasive nature and short treatment duration. SBRT delivers ablative radiation doses to smaller volumes with an intent to eradicate the low burden disease and achieve better local control and, hopefully, survival or even cure [3]. The approach for bone metastases is also dictated by the area of the skeleton that is involved. Vertebra and weight baring bone disease involvement necessitate an evaluation for the need of fixation to reduce the risk for pathological fracture. In cases of RT to treat bone metastases, the traditional RT volumes targeted the entire involved bone using palliative "conventional" doses (even if hypofractionation/single fraction was used, the total dose was relatively low) frequently resulting in suboptimal long-term local control. SBRT for spine metastasis is commonly used and is considered as one of the standard treatments for spine bone metastases in the setting of newly diagnosed disease, recurrent or progressive disease after prior RT, or post-surgery [5]. However, data on SBRT for non-spine bone metastases are scarce overall and cannot be regarded as the standard of care [6]. A few clinical series have reported excellent local control following SBRT using compound tomography (CT) or magnetic resonance (MRI) to guide treatment planning [7]. There is, however, a paucity of studies that have used molecular imaging to assess response to SBRT in the bone. Furthermore, there are no consensus guidelines on how to define the target volumes for SBRT in the non-spine bone metastases $[6,8]$.

In the current study, we evaluated ${ }^{68} \mathrm{Ga}$ PSMA PET/CT to measure local control of bone metastasis in oligometastatic prostate cancer patients treated with SBRT.

\section{Material and methods}

After the institutional review board approval, a retrospective review of medical records of consecutive prostate cancer patients treated between 2014 and 2018 was conducted. Only the medical records of patients that were treated with SBRT for oligometastatic bone lesions (defined as up to 3 metastasis) and had pre- and post-SBRT ${ }^{68} \mathrm{Ga}$ PSMA PET/CT scans were included in our study. Data extracted from the medical files included patient-related (age), disease-related (Gleason score, site of metastasis), and treatment-related factors and outcomes.

Ga 68-PSMA PET-CT scanning was performed using a combined PET-CT protocol with a 16-detector-row helical CT scanner (Gemini GXL, Phillips Healthcare). This scanner enables simultaneous acquisition of up to 45 trans-axial PET images with interslice spacing of $5 \mathrm{~mm}$ in one bed position and provides an image from the vertex to the thigh in about 10 bed positions. The trans-axial fields of view and pixel sizes of the PET images reconstructed for the fusion were $57.6 \mathrm{~cm}$ and $4 \mathrm{~mm}$, respectively, with a matrix size of $144 \times 144$. The CT component was performed with oral and intravenous contrast media. The following technical parameters were used for CT imaging: pitch 0.8 , gantry rotation speed $0.5,120 \mathrm{kVp}, 250 \mathrm{mAs}, 3-\mathrm{mm}$ slice thickness and specific breath-holding instructions. Each patient received an intravenous injection of 148 MBq Ga 68-PSMA. About 60 min later, CT images were obtained from the vertex to the mid-thigh for about $32 \mathrm{sec}$. A contrast-enhanced CT scan was obtained $60 \mathrm{sec}$ after injection of $2 \mathrm{~mL} / \mathrm{kg}$ of non-ionic contrast material (CM) (Omnipaque 370 GE Healthcare). An emission PET scan followed in $3 \mathrm{D}$ acquisition mode for the same axial image range of 2.0-2.5 min per bed position. The diagnostic CT 
images were used for the fusion with the PET data, as well as to generate a map for attenuation correction. PET images were reconstructed using a line of response protocol with CT attenuation correction, and the reconstructed images were generated for review on a computer workstation (EWB, Extended Brilliance Workstation, Philips Healthcare).

All available images were interpreted by experienced specialists in nuclear medicine and radiology and reviewed by one of the study co-authors. Ga 68-PSMA activity was quantified by calculating a maximum standardized uptake value (SUVmax). Focally increased PSMA uptake, not explained by the normal bio-distribution of PSMA, was considered positive.

Pre- and post-SBRT ${ }^{68}$ Ga PSMA PET/CT scans were reviewed and classified by a nuclear medicine and radiology specialist (YE) to determine local control at the target volume [bone enclosed by the SBRT prescription isodose line (= in-field control)]. Local control/tumor response to SBRT was classified as follows: (a) complete response (CR) - post-treatment max standardized uptake volume (SUVmax ${ }^{\text {post }}$ ) equal to the SUV mean in normal bone (SUV-NB); (b) partial response (PR) - SU$V_{\text {max }}{ }^{\text {post }}$ was less than previous SUV max (SUV$\left.\max ^{\mathrm{pre}}\right)$, but was greater than the SUV-NB (c) no Response (NR) - SUVmax ${ }^{\text {post }}$ was equal or greater than SUVmax ${ }^{\text {pre }}$.

Our department's protocol for SBRT bone metastasis includes the following: planning $\mathrm{CT}$ is performed without IV contrast with $1.0-1.5 \mathrm{~mm}$ slice thickness. Immobilization was with a Vac-Lok ${ }^{\mathrm{TM}}$ [CIVCO ${ }^{\circ}$ Radiotherapy]. For target delineation, ${ }^{68} \mathrm{Ga}$ PSMA PET/CT is fused onto the planning CT. Gross tumor volume (GTV) is delineated manually from attenuation corrected PSMA images superimposed on full dose CT, providing anatomical boundaries to the PET positive regions [9]. The clinical target volume (CTV) for vertebral lesions was segmented according to International Spine Radiosurgery Consortium (ISRC) recommendations [5]. For non-spine lesions, the CTV was a $2 \mathrm{~cm}$ expansion of the GTV around the lesion in the involved bone, no expansion into soft tissue, and without expansion for PTV. The prescription dose for this CTV was 3 fractions of 9-10 Gy delivered twice a week for 13 of the 15 lesions (Tab. 1)

RT delivery included 21iX and Trilogy machines (Varian Medical Systems, Palo Alto, CA, USA) using RapidArc (VMAT) Radiotherapy Technology guided with Cone Beam CT (CBCT) and orthogonal images prior to each fraction.

Our follow-up protocol includes a clinical evaluation every 6 months (history and physical exam) and serum PSA. Repeat ${ }^{68} \mathrm{Ga}$ PSMA PET/CT scans were performed for patients with rising PSA following SBRT in order to re-stage the patient prior to the choice of therapy, and in some cases were performed to assess response to therapy without evidence of biochemical failure.

Table 1. Patient, disease and treatment characteristics

\begin{tabular}{|c|c|c|c|c|c|c|c|c|}
\hline $\begin{array}{l}\text { PSA } \\
\text { nadir }\end{array}$ & PSA at failure & $\begin{array}{c}\text { Fx dose } x \\
\text { number of } f x\end{array}$ & $\begin{array}{l}\text { Short term } \\
\text { ADT }\end{array}$ & Lesion site & $\begin{array}{l}\text { Primary } \\
\text { therapy }\end{array}$ & Gleason & T stage & Age (yrs) \\
\hline 0.01 & 2.09 & $10 \mathrm{~Gy} \times 3$ & Yes & Ilium & XRT + ADT & 8 & T3A & 72 \\
\hline 0.06 & 6.30 & $6 \mathrm{~Gy} \times 6$ & Yes & Ilium, L5 & Surgery & 7 & T3B & 73 \\
\hline 4.32 & 21 & $10 \mathrm{~Gy} \times 3$ & Yes & D11 & XRT & 7 & T1C & 74 \\
\hline 1.52 & 3.50 & $10 \mathrm{~Gy} \times 3$ & No & Ilium, L3 & XRT & 7 & T2B & 83 \\
\hline 0.12 & 1.60 & $9 \mathrm{~Gy} \times 3$ & No & D12 & XRT + ADT & 10 & $\mathrm{~T} 2 \mathrm{~B}$ & 72 \\
\hline 0.16 & 0.65 & $10 \mathrm{~Gy} \times 3$ & No & D6 & Brachytherapy & 6 & $\mathrm{~T} 1 \mathrm{C}$ & 74 \\
\hline 1.7 & 1.48 & $10 \mathrm{~Gy} \times 3$ & Yes & D7, Ilium & Surgery & 7 & $\mathrm{~T} 2 \mathrm{C}$ & 64 \\
\hline 0.06 & 5.3 & $8 \mathrm{~Gy} \times 5$ & Yes & Pubis & Surgery & 7 & - & 76 \\
\hline 1 & 3.45 & $10 \mathrm{~Gy} \times 3$ & No & Scapula & $\mathrm{XRT}+\mathrm{ADT}$ & 9 & $\mathrm{~T} 2 \mathrm{~A}$ & 76 \\
\hline 0.01 & 44.9 & $10 \mathrm{~Gy} \times 3$ & Yes & D12 & XRT + ADT & 9 & T3A & 86 \\
\hline 0.47 & 12.6 & $10 \mathrm{~Gy} \times 3$ & Yes & D8, D10 & $\mathrm{XRT}+\mathrm{ADT}$ & 7 & $\mathrm{~T} 2 \mathrm{~B}$ & 76 \\
\hline 0.05 & 21.5 & $10 \mathrm{~Gy} \times 3$ & Yes & Rib & Surgery & 9 & T3A & 52 \\
\hline
\end{tabular}

XRT — external beam radiation; ADT — androgen deprivation therapy; Gy — Gray; PSA units — ng/mL; Fx — fraction 


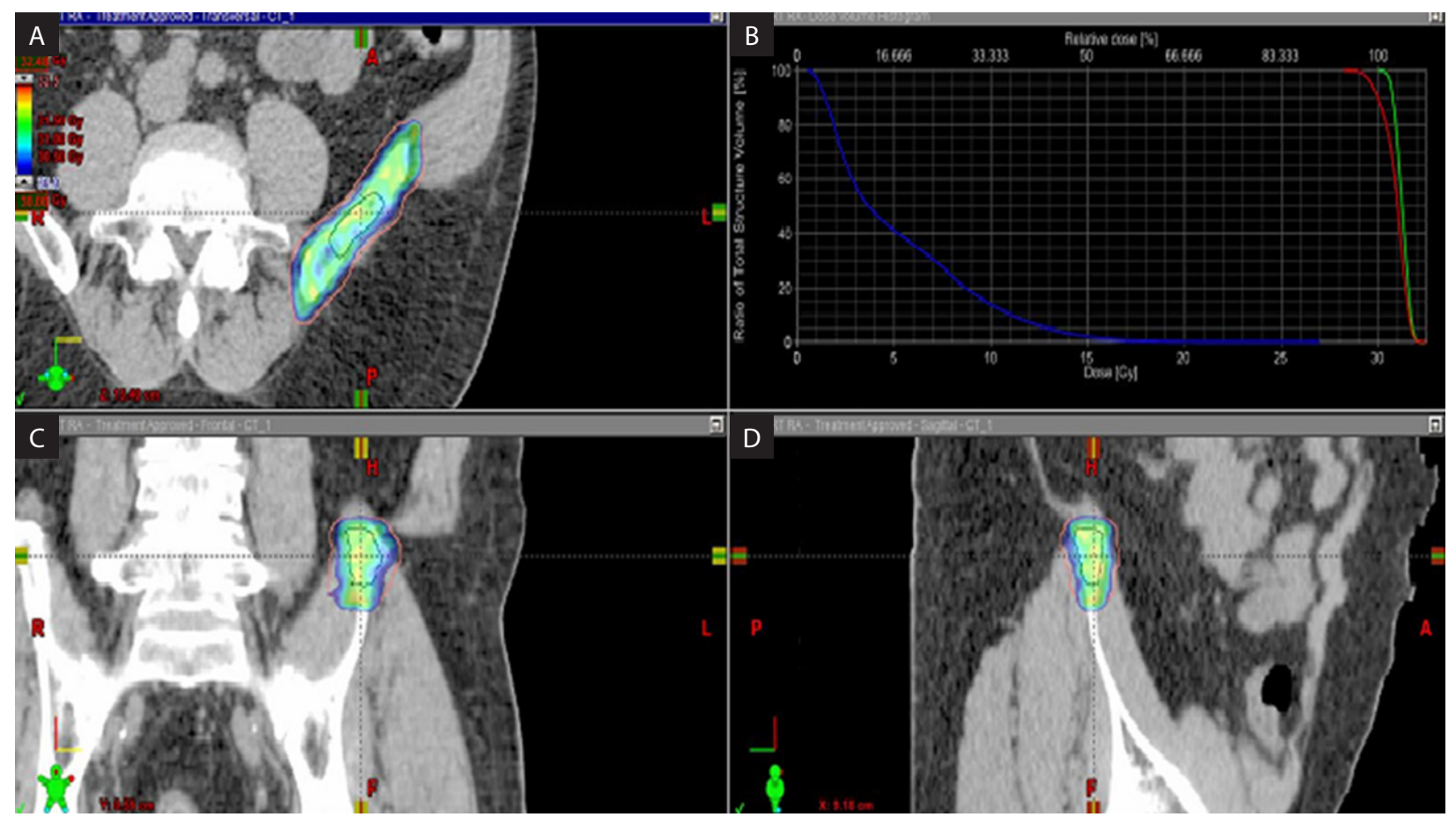

Figure 1. The plan of a right iliac lesion. A. Axial view; B. Coronal view; C. Sagittal view; D. Dose volume histogram of the stereotactic treatment plan

\section{Results}

During the study period, a total of 12 patients (15 lesions) were included, with a median age of 73 years. The median follow-up was 26.5 months (range 13-45 months). Median time of ${ }^{68} \mathrm{Ga}$ PSMA PET/CT follow up was 17 months (range 3-39 months). Patients' and treatment characteristics are summarized in Table 1. None of the patients died during the study period.

The median pre-treatment PSA was $2 \mathrm{ng} / \mathrm{mL}$ (range $0.56-44 \mathrm{ng} / \mathrm{mL}$ ) vs. post treatment PSA nadir of $0.01 \mathrm{ng} / \mathrm{mL}(0.01-4.32)$ with a median time to nadir of 7 months (range, 2-12).

Median SUVmax ${ }^{\text {pre }}$ was 5.74 (range 2.15-13.0). Complete response on repeat PSMA PT/CT was observed in 14 of 15 (93\%) lesions, with reduction in the median SUVmax ${ }^{\mathrm{pre}} \mathrm{RT}$ from 5.74 to $1.16 \mathrm{SU}$ $\mathrm{Vmax}^{\text {post }} \mathrm{RT}(\mathrm{p}<0,0003)$ (Fig. 2). One lesion had (PR) residual PSMA avidity which increased over time. Figure 3 shows repeat ${ }^{68} \mathrm{Ga}$ PSMA PET/ CT scans pre-SBRT and 3 and 6 months after radiation, demonstrating decreasing avidity over time. Out of 12 patients, 5 (41.6\%) remained free of new metastasis at 2 years, 5 developed up to 3 additional metastases at other sites (oligometastatic disease) and 2 patients developed widespread metastatic disease.
No patients developed recurrences in the treated bone. Transient grade 2 esophagitis was reported in 3 patients treated to metastasis in the dorsal spine. None of the patients had grade 3 or more toxicity related to radiotherapy during follow-up.

\section{Discussion}

Our study demonstrated an excellent outcome of SBRT for prostate cancer bone metastasis. Complete local response of the bone lesion was demonstrated by SUVmax ${ }^{\text {post }}$ equal to the mean SUV in normal bone (SUV-NB) and correlated to clinical outcome (patient's clinical evaluation).

Although our cohort is small, our study raises a few clinically relevant issues. SBRT to the bone lesions resulted in CR in $93 \%$ of the patients (similar rates were reported by Siva [10]). None of the patients failed in the treated bone (=no re-treatment), developed a fracture, or suffered from treatment related complications. At a median follow up of 26.5 months, all patients were still alive, suggesting that this patient population should be offered treatment that provides good local control. Conventional fractionation to bone metastases, as indicated by Tong et al. [11], was reported to provide CR in $49 \%$ to $61 \%$ of patients. 


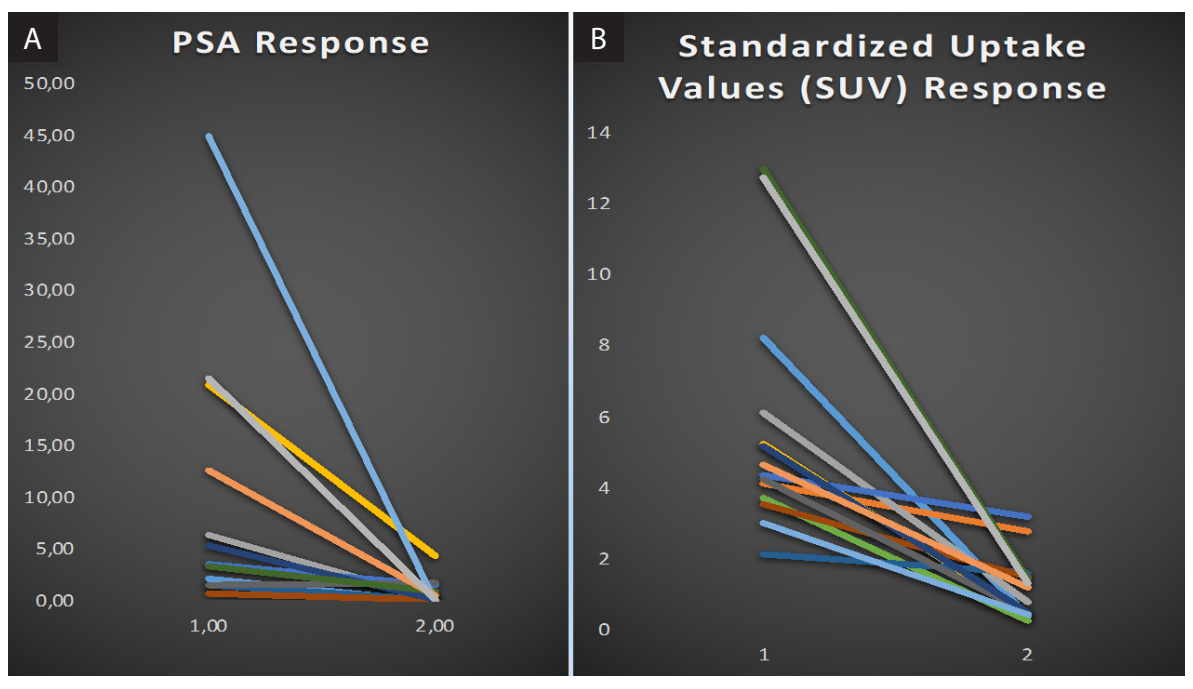

Figure 2. A. The slope of the decline in PSA (two points: pretreatment PSA and PSA nadir) following SBRT for each patient and the corresponding line in panel; B. The decline in SUVmax uptake (two points: pre/post treatment SUVmax)

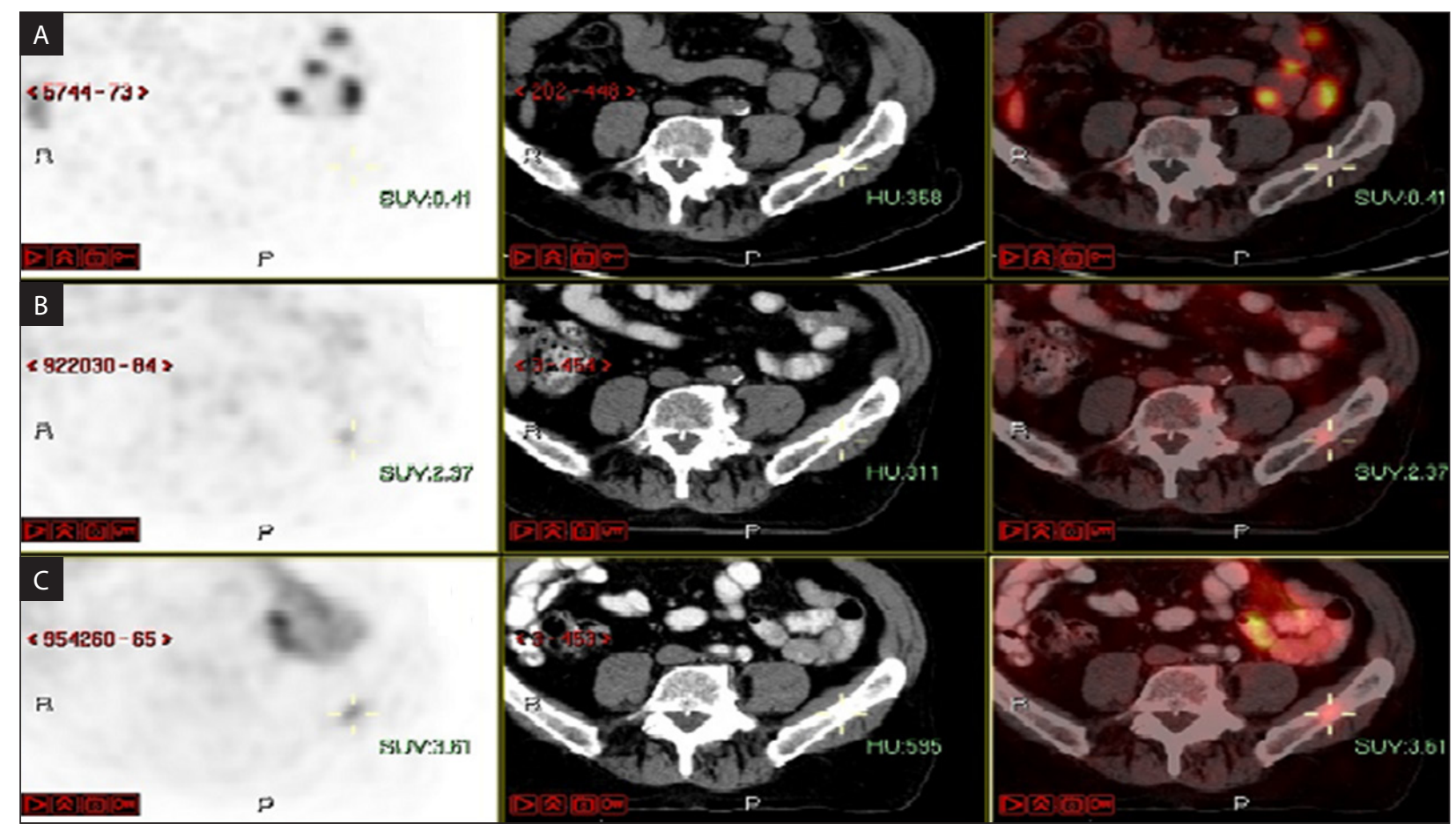

Figure 3. An example of repeat ${ }^{68} \mathrm{Ga}$ PSMA PET/CT scans. A. 6 months after radiation; B. 3 months; C. Pre-SBRT

It is difficult to compare CR results to older trials of conventional fractionation evaluating response as different definitions and imaging (if any) were used (e.g., re-treatment, pain relief). Moreover, imaging based-morphological evaluation of bone metastases can be challenging. In prostate cancer patients, the response is evaluated via PSA levels and standard CT imaging using response evaluation criteria in solid tumors (RECIST) criteria [12]. Prostate cancer bone metastases usually cause osteoblastic response which is irreversible and may or may not harbor viable cancer cells, thus, making it difficult to assess using RECIST [13]. The use of molecular imaging using ${ }^{68} \mathrm{Ga}$ PSMA PET/CT, as shown in our study, should be further evaluated as an innovative method to plan 
the target volume and evaluate response to SBRT [12].

In our study the CTV was $2 \mathrm{~cm}$ around the GTV, along the involved bone without a PTV. A $2 \mathrm{~cm}$ margin is relatively larger than what is reported in the literature $[6,8,14]$. As shown from our results, it was not associated with toxicity and could be related to the good local control and bone control. The use of ${ }^{68} \mathrm{Ga}$ PSMA PET/CT for planning was mainly for correct delineation of the GTV rather than reducing margins.

The utilization of SBRT for oligometastatic disease in prostate cancer has been studied in a number of recently published trials. The STOMP trial [15] utilized metastasis directed therapy with surgery or SBRT without androgen deprivation therapy for oligometastatic prostate cancer. Patients were randomized to local treatment or surveillance and the primary endpoint was defined as androgen deprivation therapy - free survival. The utilization of local therapy significantly delayed the need for androgen deprivation therapy which was mandated for symptomatic progression, occurrence of more than three metastases or local progression of known metastasis. The ORIOLE trial [16] randomized men with up to three metastases detected with standard imaging to observation versus SBRT delivered to all detected metastases after further evaluation with PSMA PET imaging. Progression at six months occurred in $60 \%$ of men under observation versus only $19 \%$ of men who received SBRT. Mazolla et al. [17] performed a retrospective analysis of PSMA-PET guided versus choline-PET guided SBRT, demonstrating superior control for PSMA. These studies suggest a role for PET PSMA guided metastasis directed therapy in men with oligometastatic prostate cancer._This approach may facilitate the delay of the need for androgen deprivation and, thus, perhaps be associated with improved quality of life and delay of onset of androgen independent disease. Biomarkers in the Oriole trial suggest that SBRT induced a systemic immune effect, warranting further study of this strategy.

The current study has several limitations. It is a small retrospective study with a moderate follow up of 25 months. It differs from both the STOMP and ORIOLE trials in that eight of twelve patients on study received short term androgen deprivation of 3 months concurrently with SBRT which was discontinued after the completion of radiotherapy
Thus, it is not possible to rule out a radio-sensitizing effect of concomitant ADT. This study raises important clinical issues that should be further investigated in clinical trials.

\section{Conclusions}

SBRT is a highly effective and safe method for treatment of prostate cancer bone metastases. More studies are required to determine if SBRT provides greater clinical benefit than standard fractionation for oligometastatic prostate cancer patients. ${ }^{68} \mathrm{Ga}$ PSMA PET/CT should be further investigated for delineation and follow up. Whether the optimal approach to oligometastatic recurrence is SBRT alone or in combination with short term ADT should be further evaluated.

None declared.

\section{Conflict of interest}

\section{Funding}

None declared.

\section{References}

1. Slaoui A, Albisinni S, Aoun F, et al. A systematic review of contemporary management of oligometastatic prostate cancer: fighting a challenge or tilting at windmills? World JUrol. 2019; 37(11): 2343-2353, doi: 10.1007/s00345-01902652-7, indexed in Pubmed: 30706122.

2. Kamran SC, D'Amico AV. Radiation Therapy for Prostate Cancer. Hematol Oncol Clin North Am. 2020; 34(1): 45-69, doi: 10.1016/j.hoc.2019.08.017, indexed in Pubmed: 31739952.

3. Palma DA, Olson R, Harrow $\mathrm{S}$, et al. Stereotactic ablative radiotherapy for the comprehensive treatment of 4-10 oligometastatic tumors (SABR-COMET-10): study protocol for a randomized phase III trial. BMC Cancer. 2019; 19(1): 816, doi: 10.1186/s12885-019-5977-6, indexed in Pubmed: 31426760.

4. Plouznikoff N, Artigas C, Sideris S, et al. Early Detection of Metastatic Prostate Cancer Relapse on ${ }^{68} \mathrm{Ga}$-PSMA-11 $\mathrm{PET} / \mathrm{CT}$ in a Patient Still Exhibiting Biochemical Response. Clin Nucl Med. 2020; 45(1): 81-82, doi: 10.1097/ RLU.0000000000002799, indexed in Pubmed: 31693605.

5. Cox BW, Spratt DE, Lovelock M, et al. International Spine Radiosurgery Consortium consensus guidelines for target volume definition in spinal stereotactic radiosurgery. Int J Radiat Oncol Biol Phys. 2012; 83(5): e597-e605, doi: 10.1016/j.ijrobp.2012.03.009, indexed in Pubmed: 22608954.

6. Kim EY, Chapman TR, Ryu S, et al. ACR Appropriateness Criteria $\left({ }^{\oplus}\right)$ non-spine bone metastases. J Palliat Med. 2015; 18(1): 11-17, doi: 10.1089/jpm.2014.9395, indexed in Pubmed: 25317672. 
7. Bedard G, McDonald R, Poon I, et al. Stereotactic body radiation therapy for non-spine bone metastases--a review of the literature. Ann Palliat Med. 2016; 5(1): 58-66, doi: 10.3978/j.issn.2224-5820.2015.07.01, indexed in Pubmed: 26841816.

8. Lo SS, Tseng Y, Halasz LM, Kim EY. Sine Tumors and NonSine Bone Metastases. In: Kaidar-Person O, Chen R. ed. Hyofractionated and Stereotactic Radiation Theray: A Practical Guide. Springer International Publishing, Cham 2018: 341-364.

9. Lee JA. Segmentation of positron emission tomography images: some recommendations for target delineation in radiation oncology. Radiother Oncol. 2010; 96(3): 302-307, doi: 10.1016/j.radonc.2010.07.003, indexed in Pubmed: 20708286.

10. Siva S, Bressel M, Murphy DG, et al. Stereotactic Abative Body Radiotherapy (SABR) for Oligometastatic Prostate Cancer: A Prospective Clinical Trial. Eur Urol. 2018; 74(4): 455-462, doi: 10.1016/j.eururo.2018.06.004, indexed in Pubmed: 30227924.

11. Tong D, Gillick L, Hendrickson F. The palliation of symptomatic osseous metastases final results of the study by the radiation therapy oncology group. Cancer. 1982; 50(5): 893-899, doi: 10.1002/1097-0142(19820901) 50:5<893::aid-cncr2820500515>3.0.co;2-y, indexed in Pubmed: 6178497.

12. Gupta M, Choudhury PS, Rawal S, et al. Evaluation of RECIST, PERCIST, EORTC, and MDA Criteria for Assessing Treatment Response with Ga68-PSMA PET-CT in Metastatic Prostate Cancer Patient with Biochemical Progres- sion: a Comparative Study. Nucl Med Mol Imaging. 2018; 52(6): 420-429, doi: 10.1007/s13139-018-0548-3, indexed in Pubmed: 30538773.

13. Wahl RL, Jacene $H$, Kasamon $Y$, et al. From RECIST to PERCIST: Evolving Considerations for PET response criteria in solid tumors. J Nucl Med. 2009; 50 Suppl 1: 122S-50S, doi: 10.2967/jnumed.108.057307, indexed in Pubmed: 19403881.

14. Ito K, Shimizuguchi T, Nihei K, et al. Patterns of Intraosseous Recurrence After Stereotactic Body Radiation Therapy for Coxal Bone Metastasis. Int J Radiat Oncol Biol Phys. 2018; 100(1): 159-161, doi: 10.1016/j.ijrobp.2017.08.045, indexed in Pubmed: 29066121.

15. De Bruycker A, Spiessens A, Dirix P, et al. Surveillance or Metastasis-Directed Therapy for Oligometastatic Prostate Cancer Recurrence: A Prospective, Randomized, Multicenter Phase II Trial. J Clin Oncol. 2018; 36(5): 446-453, doi: 10.1200/JCO.2017.75.4853, indexed in Pubmed: 29240541.

16. Phillips R, Shi WY, Deek M, et al. Outcomes of Observation vs Stereotactic Ablative Radiation for Oligometastatic Prostate Cancer: The ORIOLE Phase 2 Randomized Clinical Trial. JAMA Oncol. 2020; 6(5): 650-659, doi: 10.1001/ jamaoncol.2020.0147, indexed in Pubmed: 32215577.

17. Mazzola R, Francolini G, Triggiani L, et al. Metastasisdirected Therapy (SBRT) Guided by PET-CT 18F-CHOLINE Versus PET-CT ${ }^{68} \mathrm{Ga}-\mathrm{PSMA}$ in Castration-sensitive Oligorecurrent Prostate Cancer: A Comparative Analysis of Effectiveness. Clin Genitourin Cancer. 2020, doi: 10.1016/j. clgc.2020.08.002, indexed in Pubmed: 32863189. 